\title{
$\mathrm{P} 02-150$
}

\section{THE QCAE: A QUESTIONNAIRE OF COGNITIVE AND AFFECTIVE EMPATHY}

R. Reniers ${ }^{1}$, R. Corcoran ${ }^{2}$, R. Drake ${ }^{1}$, N. Shryane ${ }^{3}$, B. Völlm²

${ }^{1}$ Psychiatry, University of Manchester, Manchester, ${ }^{2}$ Psychiatry, University of Nottingham, Nottingham, ${ }^{3}$ Institute of Social Change, University of Manchester, Manchester, UK

Aims/objectives: A lack of empathy is associated with callous-unemotional behaviour, violence, aggression, criminality, and problems in social interaction. Empathy is, though, inconsistently defined and inadequately measured. We therefore set out to produce a new and rigorously developed empathy questionnaire that would have clinical and public-health relevance.

Methods: Sixty-five questions, themed around cognitive empathy (the ability to construct a working model of the emotional states of others) and affective empathy (the ability to be sensitive to and vicariously experience the feelings of others), were administered to two independent samples of healthy volunteers $(\mathrm{N} 1=640, \mathrm{~N} 2=383)$, which were used to explore and validate the factor structure.

Results: Principal components analysis revealed five factors from thirty-seven items. Confirmatory factor analysis confirmed this structure. The hypothesised two-factor structure (cognitive and affective empathy) was tested by adding two second order factors, indicated by the five first-order factors, and provided the best and most parsimonious fit to the data $(C F I=0.961, R M S E A=0.048)$. Cognitive Empathy encompassed Perspective Taking and Online Simulation; Affective Empathy encompassed Emotional Responsivity, Peripheral Responsivity and Emotional Contagion. Females scored significantly higher than males on Affective Empathy but not on Cognitive Empathy. The factors correlated significantly with measures of empathic anger, impulsivity, aggression, psychopathy, Machiavellianism and empathy as measured by the Basic Empathy Scale.

Conclusions: The QCAE measures the distinct and specific components that make up cognitive and affective empathy. The factor structure was confirmed in independent samples and represents a valid tool for assessing cognitive and affective empathy and its subcomponents. 